\title{
Near-IR photometry of southern X-ray binary systems
}

\author{
M.J. Coe ${ }^{1}$, D.A.H. Buckley ${ }^{2}$, J. Fabregat ${ }^{3}$, L.A. Steele ${ }^{4}$, M.D. Still ${ }^{5}$, and J.M. Torrejon ${ }^{3}$ \\ 1 Physics Dept., The University, Southampton, SO17 3BJ, UK \\ 2 S.A.A.O., P.O. Box 9, Observatory 7935, South Africa \\ 3 Departmento de Astronomia, Universidad de Valencia, 46100 Burjassot, Spain \\ 4 Astrophysics Group, Liverpool John Moores Univ, Liverpool, L3 3AF, UK \\ ${ }^{5}$ University of St. Andrews, St. Andrews, KY16 9SS, UK
}

Received December 5, 1996; accepted March 3, 1997

\begin{abstract}
We report IR measurements of 30 optical counterparts to a group of possible and probable High Mass X-ray Binaries (HMXRBs). In the majority of the systems these measurements represent the first reported IR flux values. In common with many other similar systems, the results show the presence of a strong, frequently variable IR signal. The implications of some of the results are discussed.
\end{abstract}

Key words: stars: binaries — X-rays: stars

\section{Introduction}

The Be/X-ray and Supergiant binary systems represent the largest sub-class of massive X-ray binaries. Of the 42 proposed massive X-ray binary pulsar systems, 35 are identified as such binaries. The orbit of the Be or supergiant star and the compact object, presumably a neutron star, is generally wide and eccentric. The optical star exhibits $\mathrm{H} \alpha$ line emission and continuum free-free emission (revealed as excess flux in the IR) from a circumstellar gas, most likely in a disk geometry.

Progress towards a better understanding of the physics of these systems depends on a multi-wavelength programme of observations. From observations of the Be star in the optical and IR, the physical conditions under which the neutron star is accreting matter can be determined. In combination with hard X-ray timing and flux observations, this yields a near complete picture of the accretion process.

As part of such a campaign, observations have been carried out of southern hemisphere objects using the SAAO $1.9 \mathrm{~m}$ telescope. The individual sources have been observed as frequently as possible in order to explore both their individual IR variability and the link with the X-ray

Send offprint requests to: M.J. Coe flux. Presented in this paper are the first IR observations of many proposed, and established HMXRB systems together with observations of a small number of sources with only one or two previously published values.

\section{IR observations}

The sources were observed with the MkIII infrared photometer (Glass 1985) on the SAAO 1.9-m telescope. A chopping secondary mirror defines two effective apertures: on-source (star) and off-source (background). Square apertures of $9^{\prime \prime}$ or $12^{\prime \prime}$ were used, depending on the seeing. A single observation module consisted of a $40 \mathrm{~s}$ integration, with the star placed alternately in the two apertures by nodding the telescope. These individual $40 \mathrm{~s}$ observations were then repeated, typically 2-4 times per filter, until a sufficient precision (standard error $\sim 0.01$ magnitudes) was achieved. The observations were obtained in the SAAO JHK system $(1.25,1.65$ \& 2.2 microns; Carter $1990)$ in photometric conditions, transformations being affected from regular observations of IR standards.

The observations were carried out during the period December 1993 - November 1995 at approximately quarterly intervals. The result from the observation of each source taken on a good photometric night, and a measure of its variability are presented in Table 1.

\section{Discussion on some individual objects}

All the sources were primarily checked against the Catalog of Infrared Observations (Gezari et al. 1994) for previous IR published fluxes. Further searches were made in the SIMBAD data base. Of the 30 sources presented here only 6 have previous reported IR measurements - GRO J100857.1, A1118-615, 1E1145.1-6141, 2S1145-619, 2S172824 and AX1845.0 - 0433 (see individual comments below for references on these objects). 
Table 1. List of sources observed in this programme. $N$ is the number of observations and $F$ is the fluctuation factor (the range of $J$ magnitudes observed divided by the number of observations)

\begin{tabular}{|c|c|c|c|c|c|c|c|c|}
\hline $\begin{array}{c}\text { Source } \\
\text { Name }\end{array}$ & $\begin{array}{l}\text { RA (2000) } \\
\text { hr mn sc }\end{array}$ & $\begin{array}{l}\text { Dec }(2000) \\
\circ^{\prime \prime \prime}\end{array}$ & Obs. date & $J$ & $H$ & $K$ & $N$ & $\bar{F}$ \\
\hline $\begin{array}{c}\text { RX J0051.8-7231 } \\
\text { 1E0050.1-7247 }\end{array}$ & 005153.4 & -723156.5 & 02 Oct. 1996 & $13.95 \pm 0.11$ & $14.06 \pm 0.19$ & $14.55 \pm 0.42$ & 1 & - \\
\hline RX J0059.2-7138 & 005912.7 & -713844.8 & 02 Oct. 1996 & $13.95 \pm 0.09$ & $14.12 \pm 0.23$ & $13.74 \pm 0.24$ & 1 & - \\
\hline SMC X-3 & 005207.7 & -722543.7 & 17 Nov. 1995 & $14.67 \pm 0.14$ & $14.78 \pm 0.45$ & $14.63 \pm 0.46$ & 6 & 0.17 \\
\hline SMC X-2 & 005434.7 & -734043.2 & 02 Oct. 1993 & $14.80 \pm 0.10$ & $15.0 \pm 0.30$ & $14.5 \pm 0.20$ & 3 & 0.09 \\
\hline $\begin{array}{c}\text { RX J005354-7226 } \\
\text { 1E0052.1-7242 }\end{array}$ & 005352.8 & -722634.4 & 14 Nov. 1995 & $13.18 \pm 0.05$ & $12.93 \pm 0.05$ & $12.84 \pm 0.06$ & 2 & 0.25 \\
\hline RX J0101.0-7206 & $\begin{array}{lll}01 & 01 & 03.2\end{array}$ & -720657 & 02 Oct. 1996 & $14.69 \pm 0.32$ & $14.15 \pm 0.43$ & $14.03 \pm 0.09$ & 1 & - \\
\hline RX J0103-722 & $\begin{array}{llll}01 & 03 & 13.9 \\
\end{array}$ & -720914.1 & 30 Jun. 1994 & $14.85 \pm 0.34$ & & & 1 & - \\
\hline $\begin{array}{l}\text { SMC X-1 } \\
\text { Sk160 }\end{array}$ & 011641.9 & -732612.4 & 14 Nov. 1995 & $13.47 \pm 0.04$ & $13.55 \pm 0.06$ & $13.43 \pm 0.15$ & 7 & 0.030 \\
\hline $\begin{array}{c}\text { RX J0501.6-7034 } \\
\text { CAL 9 } \\
\end{array}$ & 050124.5 & -703330 & 02 Oct. 1996 & $13.74 \pm 0.19$ & $12.85 \pm 0.03$ & $12.85 \pm 0.05$ & 1 & - \\
\hline $\begin{array}{c}\text { RX J0502.9-6626 } \\
\text { CAL E }\end{array}$ & 050252.5 & -662626 & 02 Oct. 1996 & $14.24 \pm 0.02$ & $14.65 \pm 0.09$ & $14.33 \pm 0.11$ & 1 & - \\
\hline EXO 0531.1-6609 & 053112.0 & -660708 & 02 Oct. 1996 & $13.99 \pm 0.06$ & $13.92 \pm 0.08$ & $13.57 \pm 0.08$ & 1 & - \\
\hline $\begin{array}{c}\text { RX J0532.5-6551 } \\
\text { Sk -65 } 66 \\
\end{array}$ & 053232.6 & -655140.8 & 03 Oct. 1996 & $13.50 \pm 0.06$ & $13.45 \pm 0.02$ & $13.60 \pm 0.14$ & 1 & - \\
\hline LMC X-4 & 053249.2 & -662214.4 & 5 Mar. 1993 & $14.58 \pm 0.10$ & $14.80 \pm 0.20$ & $14.90 \pm 0.30$ & 5 & 0.046 \\
\hline A0538-66 & 053540.27 & -665152.9 & 14 Nov. 1995 & $14.99 \pm 0.25$ & $15.08 \pm 0.36$ & $15.8 \pm 1.3$ & 1 & - \\
\hline $\begin{array}{c}\text { H0544-665 } \\
\text { Source no: } 22\end{array}$ & 054415.4 & -663350.2 & 17 Nov. 1995 & $13.73 \pm 0.09$ & $12.72 \pm 0.07$ & $12.74 \pm 0.08$ & 3 & 0.11 \\
\hline $\begin{array}{c}\text { H0739-529 } \\
\text { HD63666 }\end{array}$ & 074723.5 & -531958.3 & 14 Nov. 1995 & $7.46 \pm 0.01$ & $7.42 \pm 0.01$ & $7.41 \pm 0.01$ & 6 & 0.006 \\
\hline $\begin{array}{c}\text { 1H0749-600 } \\
\text { HD65663 }\end{array}$ & 075615.8 & $\begin{array}{lll}-61 & 05 & 59.4\end{array}$ & 14 Nov. 1995 & $6.60 \pm 0.01$ & $6.56 \pm 0.01$ & $6.55 \pm 0.01$ & 6 & 0.008 \\
\hline GRO J1008-57.1 & 100946.9 & -581735.5 & 14 Nov. 1995 & $11.57 \pm 0.03$ & $10.91 \pm 0.01$ & $10.49 \pm 0.01$ & 5 & 0.066 \\
\hline $\begin{array}{c}\text { 1E1024.0-5732 } \\
\text { Wack2134 }\end{array}$ & 102556.5 & -574841.1 & 14 Nov. 1995 & $8.88 \pm 0.01$ & $8.23 \pm 0.01$ & $7.87 \pm 0.01$ & 5 & 0.018 \\
\hline $\begin{array}{c}\text { A1118-615 } \\
\text { He3-640/Wray763 }\end{array}$ & 112052.9 & -615452.2 & 14 Nov. 1995 & $9.66 \pm 0.01$ & $9.09 \pm 0.01$ & $8.64 \pm 0.01$ & 8 & 0.009 \\
\hline $\begin{array}{c}\text { 2U1119-603 } \\
\text { Cen X-3/V779Cen } \\
\end{array}$ & 112115.2 & -603724.2 & 14 Nov. 1995 & $11.33 \pm 0.03$ & $10.86 \pm 0.01$ & $10.57 \pm 0.01$ & 4 & 0.103 \\
\hline $\begin{array}{c}\text { 1E1145.1-6141 } \\
\text { V830Cen }\end{array}$ & 114728.5 & -615713.5 & 21 Feb. 1995 & $9.71 \pm 0.01$ & $9.17 \pm 0.02$ & $8.90 \pm 0.02$ & 7 & 0.009 \\
\hline $\begin{array}{c}\text { 2S1145-619 } \\
\text { V801Cen/HD102567 } \\
\end{array}$ & $\begin{array}{lll}11 & 48 & 00.1\end{array}$ & -621224.5 & 21 Feb. 1995 & $8.66 \pm 0.01$ & $8.44 \pm 0.02$ & $8.19 \pm 0.02$ & 8 & 0.089 \\
\hline $\begin{array}{c}\text { 3U1223-624 } \\
\text { GX301-2/WRA } 977 \\
\end{array}$ & 122637.6 & -624613.2 & 23 Feb. 1995 & $6.83 \pm 0.01$ & $6.11 \pm 0.01$ & $5.72 \pm 0.01$ & 7 & 0.010 \\
\hline $\begin{array}{c}\text { 1H1253-761 } \\
\text { HD109857 }\end{array}$ & 123914.9 & -752211.8 & 22 Feb. 1995 & $6.18 \pm 0.01$ & $6.15 \pm 0.01$ & $6.11 \pm 0.01$ & 4 & 0.050 \\
\hline $\begin{array}{l}\text { GX304-1 } \\
\text { V850Cen }\end{array}$ & $\begin{array}{lll}13 & 01 & 16.4\end{array}$ & -613614.4 & 23 Feb. 1995 & $9.86 \pm 0.01$ & $9.28 \pm 0.01$ & $9.02 \pm 0.01$ & 8 & 0.012 \\
\hline $\begin{array}{c}\text { SS 2883 } \\
\text { PSR 1259-63 } \\
\end{array}$ & 130247.6 & -635119.9 .2 & 23 Feb. 1995 & $8.08 \pm 0.01$ & $7.71 \pm 0.02$ & $7.27 \pm 0.02$ & 7 & 0.013 \\
\hline $\begin{array}{c}\text { 4U1538-52 } \\
\text { QVNor/Nor X-2 }\end{array}$ & 154223.2 & -522309.9 & 22 Feb. 1995 & $10.56 \pm 0.01$ & $10.35 \pm 0.01$ & $10.14 \pm 0.01$ & 4 & 0.033 \\
\hline $\begin{array}{c}\text { 1H1555-552 } \\
\text { HD141926 }\end{array}$ & 155421.9 & -551943.6 & 22 Feb. 1995 & $7.14 \pm 0.01$ & $6.84 \pm 0.01$ & $6.51 \pm 0.01$ & 5 & 0.018 \\
\hline 2S1728-247 & 173201.6 & -244350.7 & 30 Jun. 1993 & $10.20 \pm 0.20$ & $8.72 \pm 0.03$ & $8.13 \pm 0.02$ & 7 & 0.029 \\
\hline AX1845.0-0433 & $1845 \quad 01.5$ & -043355.0 & 03 Oct. 1996 & $10.17 \pm 0.02$ & $10.61 \pm 0.02$ & $11.55 \pm 0.04$ & 8 & 0.07 \\
\hline
\end{tabular}


RX J0051.8 - 7231 - this source appears in the Bruhweiler et al. (1987) paper on new Einstein detections (identified as Source 3) and also in the ROSAT review of X-ray binary sources in the Small Magellanic Cloud (Kahabka \& Pietsch 1996). The IR flux and colours presented here are consistent with it being a Be star in the SMC.

RX J0059.2 - 7138 - this star was originally proposed as a probable Be counterpart to an X-ray binary by Hughes (1994) and confirmed by the optical spectroscopy of Southwell \& Charles (1996). These first IR measurements confirm this identification.

RX J0101.0 - 7206 - reported by Kahabka \& Pietsch (1996) as possible high mass X-ray binary system. The IR results reported here are just at the limit of the telescope, but consistent with an SMC Be/X-ray binary system.

SMC X-1, X-2, X-3 - these three sources are all well-established HMXRBs in the Small Magellanic Cloud. None of them have previously reported IR measurements and, as a group, they permit us to establish the IR charactersitics of such systems in such a location. This is particularily important when trying to identify new systems - see the two RXJ sources discussed below.

RX J005354-7226 - This object was originally proposed by Bruhweiler et al. (1987) from Einstein observations as an SMC source exhibiting a hard X-ray spectrum. Hence it was suggested as a possible Be/X-ray binary system. Subsequently a serendipitous ROSAT source, RX J005354-7226, was identified (Angelini, private communication) at a position coincident with the original Einstein position. Follow up optical and IR observations were carried out as part of this work and the IR counterpart identified. The $J-K$ colours reported here are similar to those reported in Table 1 for three other established SMC sources (SMC X -1, SMC X -2 and SMC $\mathrm{X}-3$ ) and hence support the identification of this object as being similar to them. Follow up optical spectroscopy will help identify this object better.

RX J0103 - 722 - This is one of two new Be/X-ray binary systems identified by Hughes \& Smith (1994) on the basis of ROSAT observations and $\mathrm{H}$ alpha imaging. These first IR observations confirm the detection of an IR flux but can say little more at the moment. The source at $J=14.9$ is at the limit of the telescope. Using the $V$ band measurement of Hughes \& Smith we can determine $V-J \sim 0$, similar to that of SMC X -1 .

RX J0501.6-7034 and RX J0502.9-6626 - these sources are also known as CAL 9 and CAL E, respectively (Long et al. 1981). Both re-occured in the ROSAT survey of the Large Magellanic Cloud carried out by Schmidtke et al. (1994).

EXO 0531.1 - 6609 - Reported here are the first IR observations of this proposed Be/X-ray binary system. Originally reported by Pakull et al. (1985) it was subsequently detected by ROSAT (Haberl et al. 1995a) from which pulsations at $13.7 \mathrm{~s}$ were reported (Dennerl et al. 1995).

RX J0532.5 - 6551 - Recently discovered from ROSAT data (Haberl et al. 1995b), this system could either be a supergiant or Be star binary. The one set of IR data presented here give no indication of the degree of variability and hence no clues to help resolve this ambiguity. A discussion of the properties of this source based upon optical spectra will be presented elsewhere (Coe et al. in preparation).

H0544 - 665 - This X-ray system was originally identified with another star in the region (Star 1 in the chart of Johnston et al. 1979) by van der Klis et al. (1983). The main basis for their identification was the photometric variability of the candidate. However that star exhibits no significant IR emission, unlike Star 22 reported here. Star 1 and Star 22 lie only $\sim 10^{\prime \prime}$ apart in a crowded region and it is possible that they both fell within the $9^{\prime \prime}$ aperture of the photometer used by van der Klis et al. Again, follow up optical spectroscopy should confirm this identification.

$1 \mathrm{H} 0739$ - 529 and $1 \mathrm{H0749}-600$ - Both of these HEAO-1 sources have been linked to a bright Be star in the X-ray error box (Tuohy 1988). The results presented here represent the first IR measurements of these two stars and the strong IR signal confirms their Be nature. Their association with the X-ray sources remains unconfirmed until a more accurate X-ray position is obtained, or correlated IR and X-ray variability is reported.

GRO J1008 - 57.1 - this object was identified recently by Coe et al. (1994a) as the correct optical counterpart to a new hard X-ray transient discovered by CGRO (Stollberg et al. 1993). The new IR measurements reported here demonstrate a long-term decline in the emission from the circumstellar disk from a high of $K=9.94$ at the time of the X-ray outburst.

1E 1024.0-5732 - this is the optical counterpart suggested by Caraveo et al. (1989) as the second fastest known pulsar $(61 \mathrm{~ms})$ in an X-ray emitting binary system. Those authors identified the object as an O5 type star, whereas Mereghetti et al. (1994) suggest a Wolf-Rayet interpretation and could find no evidence for the $61 \mathrm{~ms}$ pulsations. Certainly these first IR observations show a strong IR excess $(J-K) \sim 1.0$ and $(V-K) \sim 4.8$ consistent with the presence of a significant stellar wind or disk.

A1118 - 615 - this object is the counterpart to the X-ray transient system discovered in 1975 . It has only shown two major episodes of X-ray activity since its discovery, the second occuring in January 1992 (Coe et al. 1994b) at which time its IR signal was determined to be $J=9.62$ and $(J-K)=1.03$. Surprisingly, nearly 4 years later its IR signal remained virtually unchanged even though the X-ray emission has fallen below any detectable level. No other IR observations exist in the literature so it is hard to tell what the "quiescent" level is for this system. 
1E 1145.1-6141 and 2S $1145-619$ - these two systems have very similar neutron star pulse periods $(292 \mathrm{~s}$ and $297 \mathrm{~s}$ ) and are located only $15^{\prime}$ apart. Their IR behaviour, however, over the period of our observations is very different. 1E 1145.1 - 6141 (a supergiant system first measured in the IR by Ilovaisky et al. 1982) has shown no evidence of any variability. Whereas 2 S 1145 - 619 (a Be/X-ray binary first detected by Glass 1979) has varied by over 0.5 magnitude in all the IR bands. Its longterm optical and IR behaviour and their relationship to its $\mathrm{X}$-ray outbursts will be presented elsewhere (Stevens et al. 1997).

3U1223 - 624 - this system has been proposed as a hypergiant, rather than a supergiant (Kaper et al. 1995). The IR data presented here certainly confirm the existence of strong IR excess and the lack of variability is consistent with either a hypergiant or supergiant system (as opposed to a Be star). Further work is needed to resolve the hypergiant proposition.

1H1253 - 761 and $\mathbf{1 H 1 5 5 5}$ - 552 - Both of these HEAO-1 sources have also been linked to a bright Be star in the X-ray error box (Tuohy 1988). As in the case of the previous two sources discussed above $(1 \mathrm{H} 0739-529$ and $1 \mathrm{H} 0749-600)$, our results represent the first IR measurements of these two sources and the strong IR signal confirms their Be nature.

2S 1728-247 - this is system is unusual in this sample in that it contains a M6III giant in a sybiotic system. The first IR measurements of this source were presented by Glass \& Feast (1973). The data presented here indicate significant, but not very large, variations in the IR flux.

AX1845.0 - 0433 - this is the counterpart identified by Coe et al. (1996) to the ASCA hard X-ray transient reported by Yamauchi et al. (1995). The IR data reveal a steady long-term decline from $J=9.64$ to $J=10.17$ over the period July 1995 to October 1996. This is consistent with the source being the correct identification for an $\mathrm{X}$-ray object having recently undergone an outburst.

\section{Conclusions}

The first IR measurements have been presented on 24 proposed or confirmed optical/IR counterparts to X-ray binary systems. A further 6 sources have new IR measurements. Together they represent the largest coherent group of southern hemisphere, X-ray binary sources studied and provide valuable data to help in the understanding of the behaviour of these transient X-ray binary systems. One important conclusion is that the Be star systems show greater variability than the supergiant systems - the Be systems have an average $\mathrm{F}$ value of 0.052 , whereas the supergiant average is 0.030 . This may well provide a useful method of discriminating between the two systems.
Acknowledgements. We are grateful to the SAAO for the allocations of observing time and to the very helpful support of the staff. We also thank Chris Everall for helping with some of the early observations, and Paul Roche and Pablo Reig for comments on the paper. We are particularily grateful to the constructive refereeing of Dr. F. Haberl. This research has made use of the Simbad database, operated at CDS, Strasbourg, France.

\section{References}

Bruhweiler F.C., 1987, ApJ 317, 152

Carter B.S., 1990, MNRAS 242, 1

Caraveo P.A., Bignami G.F., Goldwurm A., 1989, ApJ 338, 338

Coe M.J., Roche P., Everall C., et al., 1994a, MNRAS 270, L57

Coe M.J., Roche P., Everall C., et al., 1994b, A\&A 289, 784794

Coe M.J., Fabregat J., Negueruela I., Roche P., Steele I.A., 1996, MNRAS 281, 333

Dennerl K., Haberl F., Pietsch W., 1996, Proceedings Wurzburg Conference, MPE Report 263, 131

Gezari D.Y., Schmitz M., Pitts P.S., 1994, Catalog of Infrared Observations, NASA Pub RP-1294, 3rd edition

Glass I.S., 1979, MNRAS 187, 807

Glass I.S., 1985, Irish Astron. J. 17, 1

Glass I.S., Feast M.W., 1973, Nat Phys. Sci. 245, 39

Haberl F., Dennerl K., Pietsch W., 1995a, A\&A 302, L1

Haberl F., Pietsch W., Dennerl K., 1995b, A\&A 303, 49

Hughes J.P., 1994, ApJ 427, L25

Hughes J.P., Smith R.C., 1994, AJ 107, 1363

Ilovaisky S.A., Chevalier C., Motch C., 1982, A\&A 114, L7

Johnston M.D., Bradt H., Doxsey R.E., 1979, ApJ 233, 514

Kahabka P., Pietsch W., 1996, A\&A 312, 919

Kaper L., Lamers H.J.G.L.M., Ruymakers E., et al., 1995, A\&A 300, 466

Long K.S., Helfand D.J., Grabelsky D.A., 1981, ApJ 248, 925

Mereghetti S., Belloni T., Shara M., Drissen L., 1994, ApJ 424, 943

Motch C., Pakull M., Haberl F., Dennerl K., 1996, IAU Circ. 6285

Pakull M., Brunner H., Pietsch W., et al., 1985, Space Sci. Rev. 40, 379

Schmidtke P.C., Cowley A.P., Frattare L.M., et al., 1994, PASJ 106,843

Southwell K.A., Charles P.A., 1996, MNRAS (in press)

Stevens J.B., Reig P., Coe M.J., et al., 1997, MNRAS (in press)

Stollberg M.T., Finger M.H., Wilson R.B., et al., 1993, IAU Circ. 5836

Tuohy I.R., Buckley D.A.H., Remillard R.A., Bradt H.V., Schwartz D.A., 1988, unpublished paper presented at the "Physics of Neutron, Strs \& Black Holes" Conference, Tokyo, Feb. 1988

van der Klis M., van Paradijs J., Charles P.A., et al., 1983, MNRAS 203, 279

Yamauchi S., et al., 1995, PASJ 47, 189 